This item was submitted to Loughborough's Research Repository by the author.

Items in Figshare are protected by copyright, with all rights reserved, unless otherwise indicated.

\title{
Feasibility of imaging photoplethysmography
}

PLEASE CITE THE PUBLISHED VERSION

PUBLISHER

(C) IEEE

VERSION

VoR (Version of Record)

LICENCE

CC BY-NC-ND 4.0

\section{REPOSITORY RECORD}

Hu, Sijung, Jia Zheng, V.A. Chouliaras, and Ron Summers. 2019. "Feasibility of Imaging Photoplethysmography”. figshare. https://hdl.handle.net/2134/6150. 
This item was submitted to Loughborough's Institutional Repository (https://dspace.lboro.ac.uk/) by the author and is made available under the following Creative Commons Licence conditions.

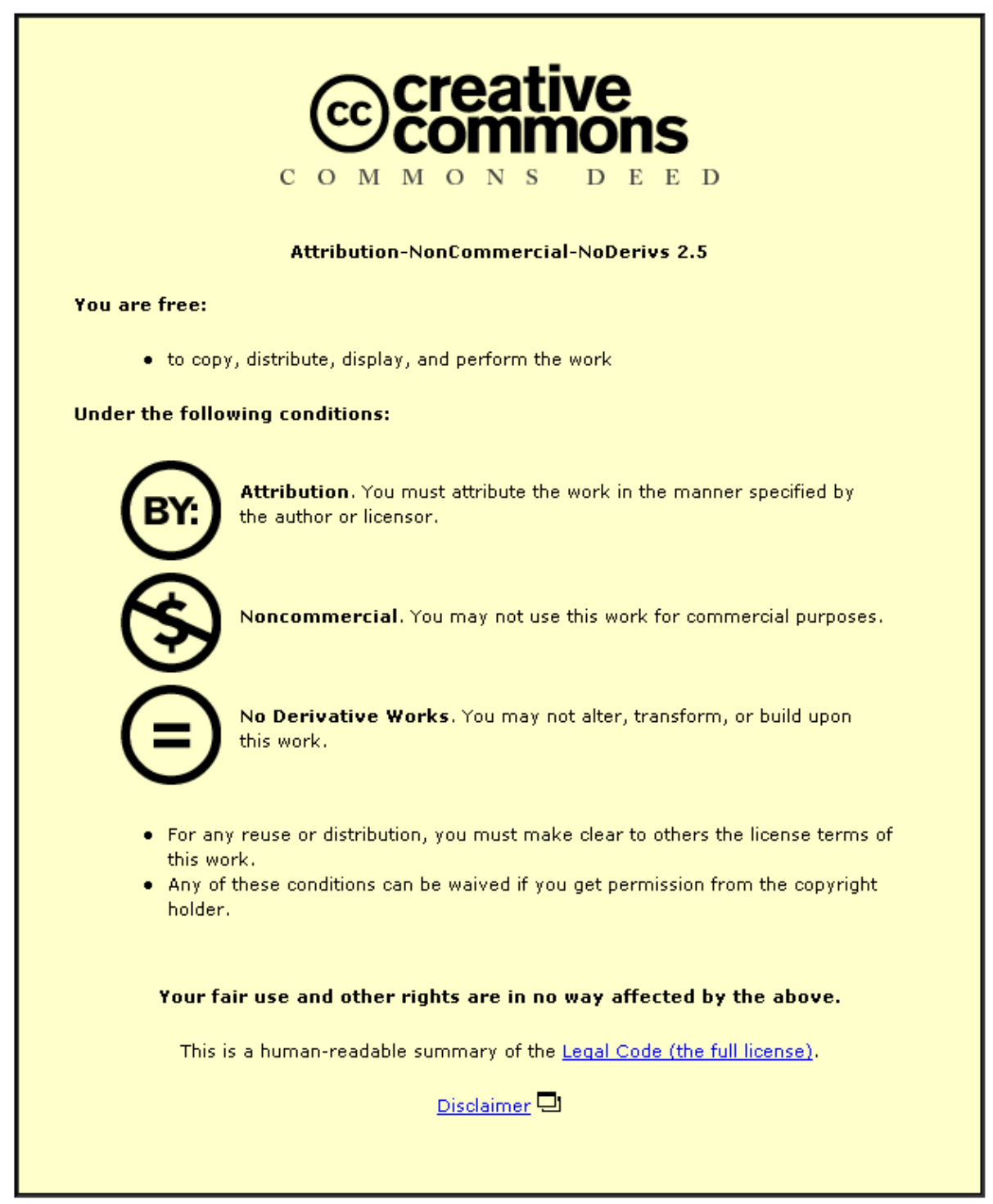

For the full text of this licence, please go to: http://creativecommons.org/licenses/by-nc-nd/2.5/ 


\title{
Feasibility of imaging photoplethysmography
}

\author{
Sijung Hu*, Member, IEEE, Jia Zheng, Vassilios Chouliaras, Member, IEEE and Ron Summers, \\ Senior Member of IEEE \\ Department of Electronic and Electrical Engineering, Loughborough University, Ashby Road, \\ Loughborough, Leicestershire LE11 3TU, UK \\ *E-mail: S.Hu@lboro.ac.uk
}

\begin{abstract}
Contact and spot measurement have limited the application of photoplethysmography (PPG), thus an imaging $P P G$ system comprising a digital CMOS camera and three wavelength light-emitting diodes (LEDs) is developed to detect the blood perfusion in tissue. With the means of the imaging PPG system, the ideally contactless monitoring with larger field of view and the different depth of tissue by applying multiwavelength illumination can be achieved to understand the blood perfusion change. Corresponding to the individual wavelength LED illumination, the PPG signals can be derived in the both transmission and reflection modes, respectively. The outcome explicitly reveals the imaging PPG is able to detect blood perfusion in a illuminated tissue and indicates the vascular distribution and the blood cell response to individual wavelength LED. The functionality investigation leads to the engineering model for 3-D visualized blood perfusion of tissue and the development of imaging PPG tomography.
\end{abstract}

Index Terms - Imaging Photoplethysmography, visualized blood perfusion, multi-wavelength illumination

\section{Introduction}

Photoplethysmography (PPG) is a non-invasive optical technique for detecting blood volume changes in tissues [1]. The fundamental modus-operandi of PPG technology is the optical detection of the dynamic cardiovascular pulse-wave, generated by the heart, as it travels throughout the body. Pulse waves obtained from the PPG signal can deliver clinically valuable information about tissue blood perfusion.

The principle of PPG, first used by Hertzman, is simple, which employs a small light source and a photosensitive detector (photoelectric cell) applied to the skin. The emitted light is scattered in the tissue and partly absorbed. Part of the scattered light emerges again through the skin and is detected by the photoelectric cell, which can be placed either beside or opposite the light source (reflection and transmission mode [3], respectively). The intensity of the light detected by reflection or transmission is converted to the PPG signal. The conventional PPG system has been limited on the single spot and contact measurement in some clinical circumstances such as wounded surface diagnosis. With the introduction of the fast digital camera into the clinical imaging monitoring and diagnosis system, the emphasis of photographic method is shifting to photonic methods that use tomography principles to non-invasive image optical contrast at depths of several millimeters to centimeters with high sensitivity [4], which inspires development of conventional PPG technology to imaging PPG. The imaging PPG which replaces the photodiode with the digital camera [5], allows to monitor larger field of view, so as to improve the ability to probe biologic interactions dynamically and to study disease over time.

In this study, a CMOS camera-based imaging photoplethysmographic system is introduced to detect the blood perfusion in specific tissue area. Attention of imaging photoplethysmography (PPG) is drawn to the potential applications in visualized blood perfusion. The imaging PPG system is presented that is capable of monitoring blood perfusion based on transmission and reflection modes, respectively, without making contact with the tissue. This study aims to develop and testify the expected experimental technique for detection of PPG signals at any selected wavelength.

\section{Experimental method}

\subsection{Sensing System}

The experimental imaging PPG system depicted in Fig. 1(a) referred the previous investigation [5]. A selected skin surface is illuminated by a multiwavelength light-emitting diodes (LED) sensor producing light centered at specific wavelengths of 660 $\mathrm{nm}, 870 \mathrm{~nm}$ and 940nm (Everylight Electronic Ltd., China), and this illuminated region is observed by a Mightex USB 2.0 color CMOS camera (Mightex. System, USA) $5 \mathrm{~cm}$ away from the measurement site. With a USB2.0 interface and the powerful PC software (Mightex System, USA), the camera delivers quality images, and the frame rate can be as high as $24 \mathrm{fps}$ in 
full resolution and up to $600 \mathrm{fps}$ using a regional of interest (ROI) mode. It has a high dynamic range of $68 \mathrm{~dB}$, acceptable quantum efficiency and a high light sensitivity, all of which make it eligible to detect the weak arterial pulsation. Depending upon the lens of the camera, it is possible to observe arbitrary parts of the skin surface, from a few square millimeter to several square centimeter as request of actual measurement.

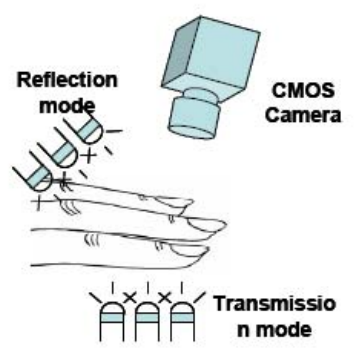

(a)

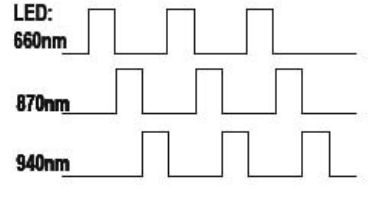

(b)
Figure 1 The experimental imaging PPG system setup as shown in figure 1(a) and triggered LEDs as shown in figure 1(b)

To compare with traditional PPG, the both transmission mode and reflection mode were explored during the experimental period. In the transmission mode, a subject's finger is illuminated by the multi wavelength LED sensor for $10 \mathrm{sec}$. In the reflection mode, the multi-wavelength LED sensor is applied on the skin surface of the subject's upper arm. The subject is requested to perform a 10 -sec spontaneous breathing and then a 10 -sec paced breathing (one deep breath in $10 \mathrm{~s})$ to test whether the imaging system can detect the breath effect on PPG signals. To reduce the effect of ambient light, the entire procedure of the measurement is operated in a optical dark room. The three wavelength LEDs are alternately trigged by a microcontroller (PIC16F876A, MicroChip Inc.) as illustrated in Fig.1(b). During the period of the measurement, the illumination light intensity from each LED is captured in alternate frames. The transmitted light intensity varies with the pulsing of the blood. A plot of this variation against time is referred to a photoplethysmography (PPG). A frame rate of 90 frames per second for the three wavelength LEDs illumination ( 30 frames per wavelength per second) is corresponding to a $120 \times 160$ pixel resolution, which can provide an appropriate trade off between two sampling intervals. This procedure makes the detection enough to capture the fine structure of the PPG signals and allow the camera sufficient time to integrate and read out each frame. When the measurement initiates, the camera records the captured frames to a PC as a set of uncompressed JPEG files.

\subsection{Data Analysis}

The resulting frames from the camera are processed by a custom algorithm written by Matlab (The Math Work Inc. USA). Each frame is divided into boxes or groups of adjacent pixels as shown in Fig. 2(a). In each frame the average pixel value occurred is calculated in a designated box. Plotting the average pixel value of each box from each frame against the time yields a PPG signal as shown in Fig. 2(b). The variations of the pixel values in each frame are influenced by the change of the finger absorption as the blood pulsate varies. The change in the ambient light is sensitively impact the detection of the camera. Although the camera is more susceptible to interference from ambient light than a conventional contact probe, the PPG signals captured by the camera are shown that is comparable to those captured by conventional probe. After the filtration of noise and the DC component, a characteristic PPG AC signal can be derived in both time domain and frequency domain as shown in Fig 2 (c), and (d) respectively. While collecting the PPG signals from all the regions in a selected image, the mean AC amplitude and DC value of individual regions over the measurement period are derived to express regional blood perfusion situation.

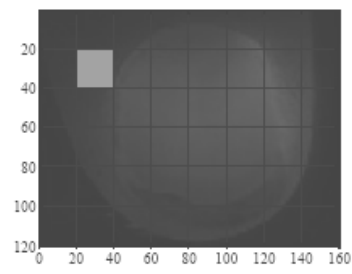

(a)

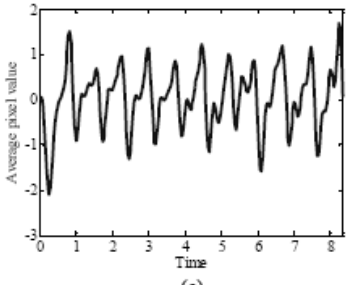

(c)

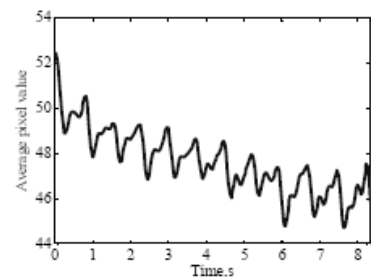

(b)

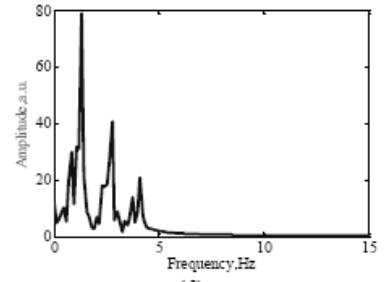

(d)
Figure 2 The procedure of data analysis to derive PPG signals. Fig. 2(a) shows one of the imaging frames is divided into boxes of adjacent pixels and the average pixel value of each box is calculated. Plotting the average pixel value of each box against the time yields a PPG signal as shown in Fig. 2(b). A time domain PPG AC signal and a frequency domain PPG signal as shown in Fig. 2(c) and Fig 2(d) respectively.

\section{Results}

\subsection{Transmission mode}

Fig. 3 depicts the results of a perfusion acquisition obtained from the camera-based PPG transmission 
system. Fig. 3(a) shows the PPG signals of marked ROIs illuminated by the 870nm LED. The PPG signals of the same ROI from 3 wavelength LEDs illumination are compared in Fig. 3(b).

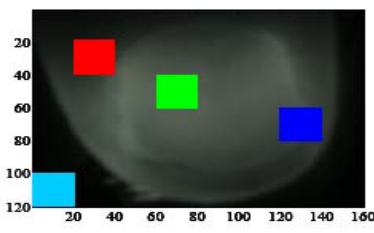

(a)

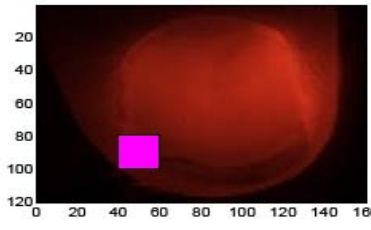

(c)
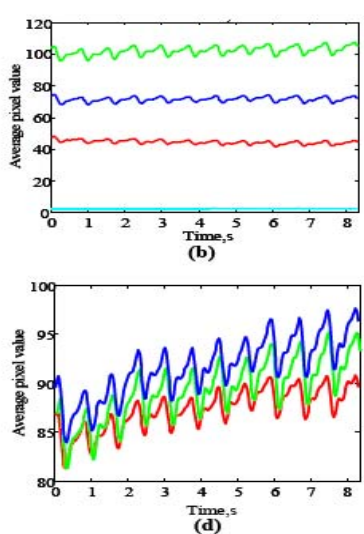

Figure 3 The results of a perfusion acquisition obtained from the camera-based PPG transmission system. Fig. 3(a) shows four marked ROIs are illuminated by the $660 \mathrm{~nm}$, $870 \mathrm{~nm}$ and $940 \mathrm{~nm}$ LEDs respectively. The PPG signals of the selected ROI from 3 wavelength LEDs illumination are compared in Fig. 3(b), in which the blue signal is from $940 \mathrm{~nm}$ illumination, the green one from $870 \mathrm{~nm}$, and the red one from $660 \mathrm{~nm}$. Fig. 3(c) exhibits one marked ROI is illuminated by the $660 \mathrm{~nm}, 870 \mathrm{~nm}$ and $940 \mathrm{~nm}$ LEDs respectively. Fig. 3(d) displays the PPG signals from 3 wavelength LEDs in the same illuminated area.
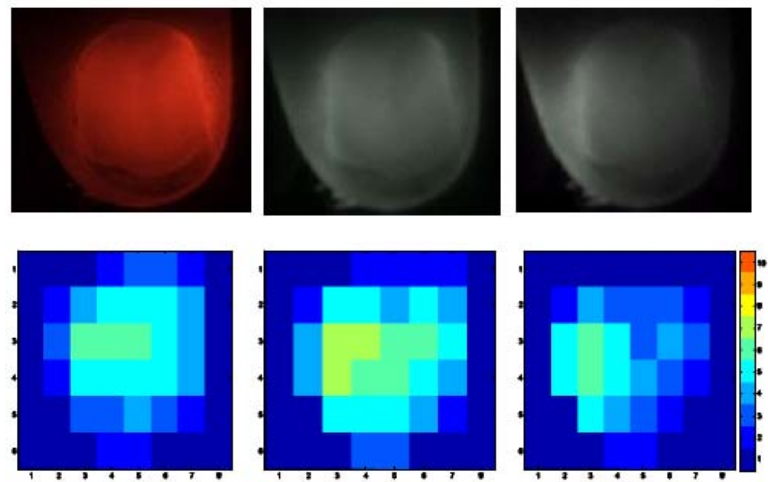

(a)

(b)

(c)

Figure 5: The whole blood perfusion of the transilluminated finger. (a) for 660nm LED; (b) for $870 \mathrm{~nm}$; and (c) for $940 \mathrm{~nm}$.

Based upon the mean AC amplitude of individual Fig. 3 depicts the results of a perfusion acquisition obtained from the camera-based PPG transmission system.Based upon the mean AC amplitude of individual

regions, the blood perfusion of the trans-illuminated finger can be derived as shown in Fig. 4 from each wavelength LED illumination.

\subsection{Reflection mode}

Fig. 6 depicts the result of the marked region from the imaging PPG reflection system. Because the arterial pulsation is relatively so weak comparing to the large DC component in the upper arm, it is difficult to separate PPG AC component from DC.
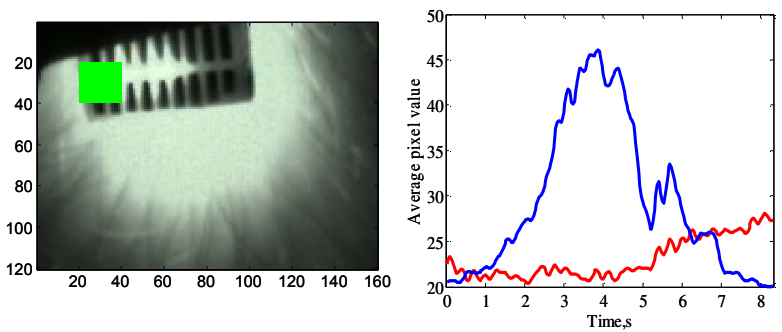

Figure 6: The result of the marked region from the imaging PPG reflection system with 870nm LED illumination in two respiration modes. The red signal is from normal breathing, and the blue one is from controlled breathing.

Similarly, based on the mean DC value over the test period, the blood perfusion can be visualized as Fig. 7 . The perfusion from different wavelength LEDs is different, which is caused by the different penetration depths of different wavelength LEDs, and thus the illumination of different vascular beds [2].

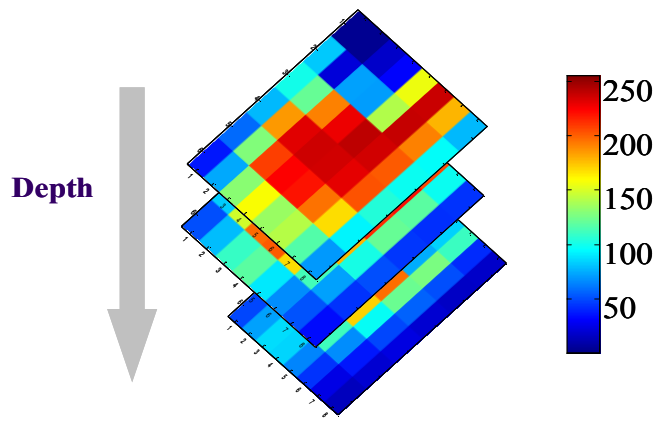

Figure 7: The blood perfusion of the whole measurement site from the imaging PPG reflection system from 3 wavelength LEDs. The top one is from $660 \mathrm{~nm}$; the middle one from 870nm; and the bottom one from $940 \mathrm{~nm}$.

\section{Discussion}

The PPG signals derived from different ROIs in Figure 5 shows their difference in both shape and amplitude. Also the PPG signals are contributed from selected area containing specific tissue components and skin vascular distribution. For the same ROI, the PPG signals are also different, for the response of blood cells to individual wavelength LEDs is different.

In the refection mode, since the arterial pulsation in the illuminated tissue area was quite weak, the PPG AC amplitude appeared smaller than in the 
transmission mode as shown in Figure 6. However the DC component can still represent many typical characters of tissue blood perfusion. From the results from two respiration modes: spontaneous breathing and paced breathing, the respiration effect on DC signal is quite obvious, which is consistence with the response of traditional PPG signal. As the mean DC value over the test period, the blood perfusion in the illuminated tissue are can be visualized by this means of the data processing.

\section{Conclusion}

The newly developed imaging PPG system has been investigated. The PPG signals obtained from the camera-based system are quite comparable to those from traditional PPG. The resulting signals demonstrated the feasibility of this technique, which allows us to study blood perfusion in 3-D visualization.

The imaging PPG system will bring significant benefits to assess large field measurement of segment blood perfusions in a selected tissue. The functionality of the system leads to the further development of the engineering model to separate the blood perfusion of individual segments in tissue, then the 3-D description of blood perfusion will be visualized.

\section{Acknowledgement}

The authors are grateful to our eternal supervisor Professor Peter Smith, and would like to thank all the members in the Photonics Engineering and Health Technology Research Group of Loughborough University for their enthusiastic support.

\section{References}

[1] A. B. Hertzman, "The blood supply of various skin areas as estimated by the photoelectric plethysmography," AM. J. physiol., 1938, vol. 124, pp. 329-340.

[2] L. G. Lindberg \& P. A. Oberg., "Photoplethysmography. Part 2. Influence of light source wavelength," Med. Biol. Eng. Comput., 1991, vol. 29, pp. 48-54.

[3] J. A. Nijboer, J. C. Dorlas, and H. F. Mahieu, "Photoelectric plethysmography-some fundamental aspects of the reflection and transmission method," Clin. Phys. Physiol. Meas., 1981, vol. 2, No. 3, pp. 205-215.

[4] V. Ntziachristos, J. Ripoll, V. L. Wang and R. Weissleder, "Looking and listening to light: the evolution of whole-body photonic imaging," Nature Biotechnology, 2005, vol. 23, No. 3, pp. 313-320.

[5] Zheng J. and Hu S., "The preliminary investigation of imaging photoplethysmographic system," IoP Publishing, 2007, pp.012031, ISBN: 1742-6588 